\title{
Equal Strength and Equal Stress Structures. Models and Reality
}

\author{
Vladimir I. Andreev ${ }^{1,2, a^{*}}$, Ivan A. Potekhin ${ }^{3, b}$ \\ ${ }^{1}$ Moscow State University of Civil Engineering (National Research University), \\ 26 Yaroslavskoye Shosse, Moscow, 129337, Russia \\ ${ }^{2}$ Scientific Research Institute of Building Physics, 21 Lokomotivny pr., Moscow, 127238 Russia \\ ${ }^{3}$ Kostroma State Agricultural Academy, Kostroma region, settl. Karavaevo, 156530, Russia \\ aasv@mgsu.ru, biv.potehin@yandex.ru
}

Keywords: Strength, Stresses, Inverse Problem, Inhomogeneity, Optimization

\begin{abstract}
The paper discusses the concepts of equal strength and equal stress structures. We will call equal strength structure, if at each point the equivalent stress corresponding to a particular theory of strength is equal to the ultimate strength of the material. In equal stress structure at all points of the body the stress state is the same. Considering that in inhomogeneous bodies ultimate strength may be variable equal strength and equal stress structures are different concepts. On the basis of the inverse problem of elasticity theory of inhomogeneous bodies and strength criteria for the concrete is considered the method of constructing the model equal strength thick-walled concrete cylinder. In practice, the creation of a concrete cylinder with continuous inhomogeneity is quite difficult. Therefore, there is proposed a method of creating a piecewise inhomogeneous structures are close to equal strength.
\end{abstract}

\section{Introduction}

Hard to imagine construction that when the loads reach limit values have destroyed at all points simultaneously. In terms of solid mechanics, this means that an equivalent stress corresponding to the theory of strength for a given material must be the same at all points of the body. The simplest example of such a structure is stretched or compressed rod (Fig. 1(a)), for which according to the hypotheses adopted in the strength of materials, at all points of the normal stresses are constant and equal $\sigma=P / A$. However, in this solution according to the principle of Saint-Venant's not considered features of arising in the place of loading and fastening. In laboratory experiments to determine the mechanical properties of materials tested rods in which there is a so-called working area in which there is a uniform stress state described by the above formula. Nevertheless, when a force $P$ reach the limit value destruction occurs in a local area (Fig. 1(b)), where a cross section is weakened, or material strength below. We shall assume that the rod shown in Example is equal stress structure, We shall assume that the rod is shown in example equal stress structure, wherein the stress at all points including a point close to the fracture, if not identical, as close as possible. Equal stress structure is not always equal-strength. This requires that not only equivalent stresses at all points of the body were the same, but tensile strength was also constant. If we imagine a rod which consists of two sections from different materials (Fig. 1(c)), it will be equal stress, but will not be uniform strength - destruction will take place at the part of the rod, which material has a lower tensile strength. Another well-known example is the beam of equal resistance in bending, shown in Fig. 2. This construction is often called equal-strength, although according to the above definition, it is not. In the beam maximum stresses in each section are the same, i.e. all sections (but is not all beam as a whole) ideally reach the limit state simultaneously. And, of course, this beam is not equal stress because stresses vary in height cross-sections. 




Fig. 1 Stretched rod.



Fig. 2 Beam of uniform strength.

\section{Construction of the Model of Equal Strength Cylinder}

For the example of Lame problem for thick-walled pipe (Fig. 3(a)), we consider the method of creation equal strength and equal stress structures based on the solution of inverse problems of the theory of elasticity of inhomogeneous bodies.
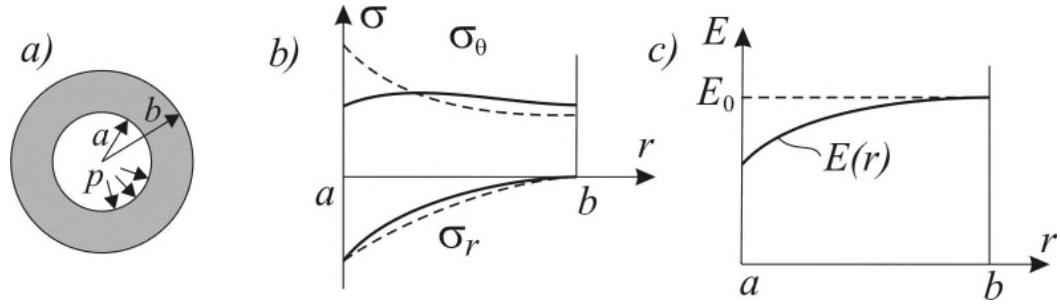

Fig. 3 The Lame's problem for thick-walled pipe .

We explain the basic idea of the method. Fig. 3(b) shows the qualitative stress distribution (dotted) in the pipe loaded internal pressure where the material is homogeneous, i.e. modulus $E=E_{0}=$ const (dotted line in Fig. 3(c)). If, for simplicity, we assume that for the material is valid the strength theory of maximum normal stress, then when the pressure $p$ reach limit the destruction begin in the internal contour of pipe where $\sigma_{\theta}=\sigma_{\max }$. At the same time the rest of the pipe is "underloaded".

From the theory elasticity of inhomogeneous bodies [1] it is known that in inhomogeneous bodies in the areas where the elastic modulus is less stresses reduced compared to the homogeneous material and vice versa. In figure $3 \mathrm{~b}$ the solid line shows the qualitative diagram corresponding to a solution to pipe, wherein the modulus is a function $E=E(r)$ (Fig. 3(c)). Note that the effect of inhomogeneity has little effect on the stresses $\sigma_{r}$ and the curve $\sigma_{\theta}$ changes substantially approaching the constant.

In [2-5] are considered certain problems for finding such dependences $E(r)$, in which the equivalent stresses corresponding to the various theories of strength will be constant. This so-called inverse problems, in which are sought the function changes the modulus of elasticity for a given stress state of the structure. For example, if for the material is valid the theory of maximum shear stresses then under the condition that in the pipe $\tau_{\max }=\left(\sigma_{\theta}-\sigma_{r}\right) / 2=$ const, this function has the form.

$$
E(r)=E_{0}\left[\frac{\left(p_{a}-p_{b}\right)(1+k \ln r)+k\left(p_{b} \ln a-p_{a} \ln b\right)}{\left(p_{a}-p_{b}\right)(1+k \ln a)+k\left(p_{b} \ln a-p_{a} \ln b\right)}\right]^{\frac{2}{k}}
$$

In (1) $k=(1-2 v) /(1-v)$ for plane strain, $k=(1-v)$ for plane stress (thin disk), and $v$ Poisson's ratio.

Note that the model obtained solution corresponds to the equal stress pipe (or disk), since at this stage is not considered strength properties of material. 
The question of creating a real equal stress and equal strength structures will be discussed below. To modulus of elasticity changed we must find some way to change the structure the material. One possibility is the use of composite materials in which by changing the percentage of filler can change their deformation properties. However, simultaneously with the change in hardness, often changing and strength of material. This implies that the ability to turn equal stress structure in equal strength can be realized only in case if the modifying of material structure its mechanical properties a lesser degree than deformational.

Table 1 shows an example of such a material [6], which corresponds to said requirement.

Table 1. Mechanical properties of polymer concrete filled with silica flour.

\begin{tabular}{|c|c|c|}
\hline $\begin{array}{c}\text { Degree of admission } \\
\text { silica flour }\end{array}$ & $\begin{array}{c}\text { Modulus elasticity } \\
E_{b}, 10^{-4} \mathrm{MPa}\end{array}$ & $\begin{array}{c}\text { Compressive strength, } \\
\mathrm{MPa}\end{array}$ \\
\hline- & 3,10 & 142 \\
\hline 50 & 4,50 & 146 \\
\hline 100 & 7,10 & 160 \\
\hline 200 & 10,5 & 148 \\
\hline 300 & 13,7 & 132 \\
\hline 400 & 16,7 & 115 \\
\hline
\end{tabular}

If you change the polymer elastic modulus of more than five times the tensile strength varies non-monotonically, varying in the range of $40 \%$. Analogous properties also have cement concretes [7]. We give an example of creating mechanical model equally strength concrete cylinder made of polymer using the strength criterion P.P. Balandin [8]. Assuming on the basis of [9] that near the time of destruction $v=0.5$ strength condition can be written as:

$$
0.75\left(\sigma_{r}\right)^{2}-1.5 \sigma_{r} \sigma_{\theta}+0.75\left(\sigma_{\theta}\right)^{2}+0.5 R_{b}\left(\sigma_{r}+\sigma_{\theta}\right)=0
$$

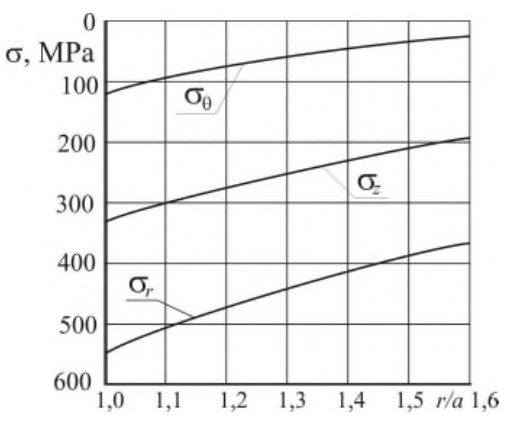

Fig. 4 Diagrams of stress in equal strength cylinder.

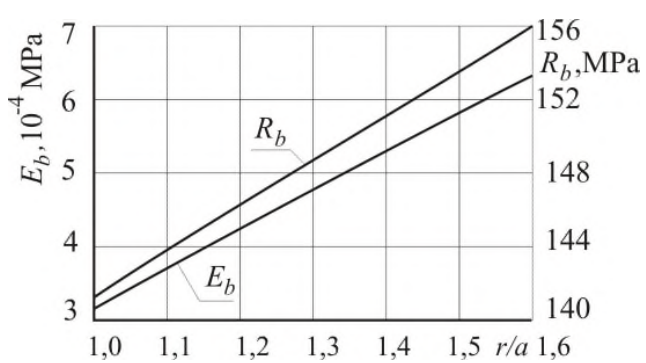

Fig. 5 Changes in elastic modulus and prism strength.

where, $R_{b}$ - prism strength of concrete. Using approximating dependence $R_{b}=\alpha+\gamma E_{b}$ built on the basis of experimental data, and using parametric method of solving Eq. (2) we can obtain the solution of the inverse problem of the theory of elasticity of inhomogeneous bodies [3]. Fig. 4 shows the stress distribution in the cylinder, obtained for the following initial data: the ratio of external and internal radii $-b / a=1.6$, the ratio of internal and external pressures $p_{a} / p_{b}=1.5, E_{b}^{(0)}=3.1 \cdot 10^{4} \mathrm{MPa}$, $R_{b}^{(0)}=141 \mathrm{MPa}, \alpha=127.7 \mathrm{MPa}, \gamma=4.61 \cdot 10^{-4}$. Fig. 5 shows dependences of $E_{b}(r)$ and $R_{b}(r)$ corresponding to the model equal strength cylinder. It should be noted that the elastic modulus varies in thickness of the pipe about 2 times, and a tensile strength - $10 \%$ only.

The obtained model of equal strength inhomogeneous cylinder can be compared differently with the corresponding homogeneous structure, for example, comparing the thickness of the cylinder wall with the same loads, or comparing the value of maximum loads in the two cases. Let us introduce the concept of the coefficient of efficiency of the equal strength cylinder as follows: 


$$
\beta=p_{a}^{(\mathrm{nh})} / p_{a}^{(\mathrm{h})}
$$

where, $p_{a}^{(\mathrm{nh})}$ and $p_{a}^{(\mathrm{h})}-$ correspondingly limit pressures in inhomogeneous and homogeneous cylinders. In the above example $p_{a}^{(\mathrm{nh})}=549.1 \mathrm{MPa}$. From Lame problem solutions for the homogeneous cylinder with the same input data can be found $p_{a}^{(\mathrm{n})}=213.5$ Мпа from which we obtain $\beta=2.57$.

\section{On Ways of Practical Implementation}

It is obvious enough that the model equally strong cylinder is difficult to realize in practice. In this connection, it is proposed to approximate continuous functions $E_{b}(r)$ and $R_{b}(r)$ on piecewise constant dependences. In other words, is invited the creation of a thick-walled cylinder consisting of several layers. In calculating such layered cylinder in each layer will be a solution of the problem Lame for a homogeneous material with their constants, which are determined by the boundary conditions on the inner and outer surfaces of the cylinder and at the boundaries of layers:

$$
\begin{array}{lll}
r=a, & \sigma_{r}=-p_{a} ; & r=b, \quad \sigma_{r}=-p_{b} ; \\
r=r_{i}, & \sigma_{r, i-1}=\sigma_{r, i} ; & u_{i-1}=u_{i},
\end{array}
$$

where, $r_{i}$ - radii of borders between layers.

The fundamental issue is the selection of the values of the elastic modulus in each layer. At first glance, one should choose the average value of $E_{i}$ in the interval $\left[r_{i-1}, r_{i}\right]$, however, the equivalent stresses in each layer exceed the corresponding values $R_{b}(r)$ obtained for the continuous dependence $E(r)$. In order to satisfy the condition of strength at all points of the cylinder must be set value $E_{i}$ corresponding to the value at the left edge of each layer.

Calculations of three-, four- and five-layer cylinders were held. Fig. 6 shows the stress distribution in a three-layer cylinder, and Fig. 7 - comparatives dependences of equivalent stresses and function $R_{b}(r)$ in the equal strength cylinder.

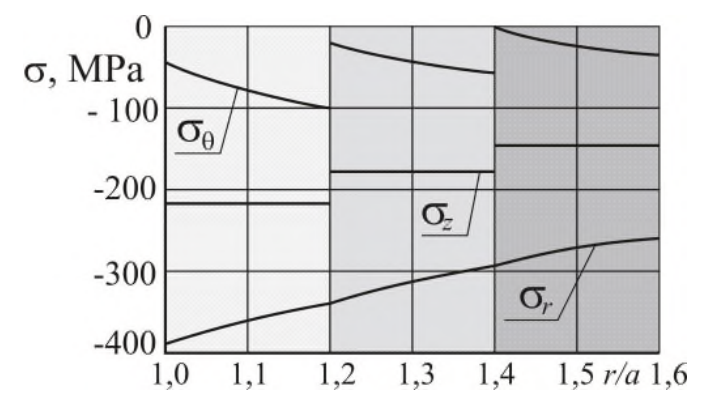

Fig. 6 The stress distributions in a three-layer cylinder.

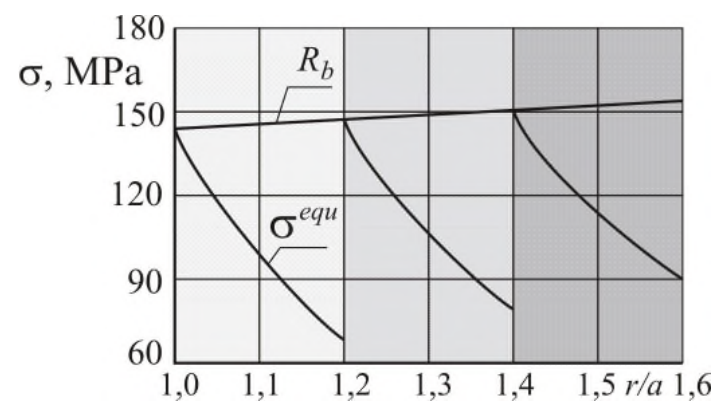

Fig. 7 The equivalent stresses in three-layer cylinder.

It is obvious enough that in a piecewise homogeneous cylinder efficiency factor is less than a cylinder with a continuously varying modulus. Calculations have shown that for the three-layer cylinder $\beta^{(3)}=1.83$. Accordingly, for the four- and five-layer cylinders $\beta^{(4)}=1,99$ and $\beta^{(5)}=2,09$. With increasing the number of layers increases the value of $\beta$ approaches the value $\beta=2.57$ corresponding to equal strength cylinder/ 


\section{Conclusions}

The paper shows that the terms of equal strength and equal stress structures are not synonymous. The example shows that the structure can be equal strength, but not equal stress, and vice versa. In many cases you cannot at all create a model equal stress design (example - a beam of equal resistance to bending).

\section{Acknowledgement}

This work was financially supported by the Ministry of Russian Education (state task \#7.1524.2017/PCh)

\section{References}

[1] V. I. Andreev. Some problems and methods of mechanics of inhomogeneous bodies. Publishing house ASV, 2002.

[2] V. I. Andreev, I. A. Potekhin. On a method to create optimal designs based on the solution of inverse problems of the theory of elasticity of inhomogeneous bodies. RAASN, Herald Building Sciences Branch, (2007), pp. 48-52.

[3] V. I. Andreev, Y. N. Malashkin. Calculation of thick-walled pipes made of nonlinear elastic material. Build. Mech. Calculat. Constr. 6 (1983) 70-72.

[4] V. I. Andreev, I. A. Potekhin. Modelling of equal strength cylinder based on an iterative approach. Int. J. Comput. Civil Struct. Eng. 4(1) (2008) 79-84.

[5] A. S. Chepurnenko, V. I. Andreev, B. M. Yazyev. Construction of a model of equal stress cylinder based on the theory of Mohr strength. Herald MSUCE. 5 (2013) 56-61.

[6] V. V. Paturoev. Polymer concrete. Moscow, Stroyizdat, 1987.

[7] G. A. Smolyago. On the question of ultimate extensibility of concrete. Concr. Reinf. Concr. 6 (2002) 6-9.

[8] G. A. Geniuses, V. N. Kissyuk. On the question of a generalized theory of the strength of concrete. Concr. Reinf. Concr. 2 (1965) 16-19.

[9] N. I. Karpenko. General models of concrete mechanics. Moscow, Stroyizdat, 1996. 